Apidologie, 1974, 5 (3), 289-293.

\title{
EINE METHODE ZUR REGISTRIERUNG DES AUS- UND EINFLIEGENS EINZELNER BIENEN
}

\author{
Méthode d'enregistrement des sorties et des rentrées \\ d'abeilles individuelles \\ Bernhard und Rutger VERBEEK \\ Aus dem Biologie-Seminar der Pädagogischen Hochschule Ruhr \\ (Seminardirektor Prof. Dr. C. Ullrich)
}

\section{SUMMARY}

A METHOD FOR RECORDING THE ARRIVAL AND DEPARTURE

FLIGHTS OF INDIVIDUAL BEES

A method is described allowing the automatic, selective recording of individual bees when leaving the hive and returning to it. The bees, marked on the thorax by a reflex folium, are counted photo-electronically, their inbound and outbound flights registered by a time interval recorder. For the time being, the method was tested in investigations of honeybee queens' mating flights.

\section{ZUSAMMENFASSUNG}

Es wird eine Methode beschrieben, die es gestattet, bestimmte Honigbienen selektiv beim Ein - und Aus fliegen automatisch zu registrieren. Die auf dem Thorax mit Reflexfolie markierten Individuen werden photoelektronisch erfaßt, ihr Aus - und Ein fliegen auf einem Zeitmarkenschreiber registriert. Erprobt wurde diese Methode bisher beim Studium der Paa rungs flüge von Königinnen.

Der Bienenstaat bietet eine Reihe Probleme, die sich nur lösen lassen, wenn das Ein - und Aus fliegen bestimmter Individuen registriert wird. Von der Möglichkeit der individuellen Markierung macht fast jeder Imker bei seinen Königinnen Gebrauch. Nach demselben oder einem abgewandelten 
Verfahren werden natürlich für bestimmte Zwecke auch Arbeiterinen und Drohnen markiert.

Will man das Verlassen des Stockes und das Wiedereintreffen für solche Bienen registrieren, so genügt die direkte Beobachtung des Fluglochs. Diese erfordert aber - wenn der Versuch über längere Zeit läuft und mehrere Stöcke überwacht werden sollen - einen enormen Personalaufwand, der selten verfügbar und kaum zu rechtfertigen ist.

Bei dem hier beschriebenen Verfahren erfolgt die Beobachtung durch einen Phototransitor und die Aufzeichnung durch einen Zeitmarkenschreiber.

Der Gedanke der photoelektronischen Überwachung ist nicht neu. Jüngst (Juni 1973) berichteten Burrill und DiETz (s. dort weitere Literatur) im American Bee Journal über den Einsatz einer Lichtelektronik zur Kontrolle der Flugaktivität eines Bienenvolkes. Sie trennten durch ein geeignetes Klappensystem Ein - und Augsang; somit konnten Ein - und Ausflüge gesondert registriert werden. Erstmalig setzte, laut obengenannten Autoren, 1933 Brittaiv eine Photoelektronik in der Bienenkunde ein. Soweit bekannt, sind aber alle bisher beschriebenen Apparate, die auch unter anderer Fragestellung entwickelt wurden, nur zur Registrierung der Gesamtaktivität geeignet und nicht in der Lage, ausgewählte Individuen zu unterscheiden. Allenfalls kann man die Zählung auf Arbeiterinnen beschränken, wenn man ein Gitter einsetzt. Bei einem gesonderten Drohnen - oder Königinnengang, läßt sich aber nicht verhindern, daß dieser auch von Arbeiterinnen benutzt wird. Das folgende Verfahren erlaubt eine selektive Registrierung markierter Individuen, die man in beliebiger Anzahl entsprechend der jeweiligen Fragestellung auswählen kann.

Der Phototransistor ist in der Mitte eines Scheinwerfers (Taschenlampe mit 0,6 W-Birne) angebracht, der auf eine Stelle gerichtet ist, die die Bienen zum Verlassen des Stockes passieren müssen (Abb. 1). Die zu untersuchenden Bienen werden mit Scotchlite, einer reflektierenden Folie, auf dem Thorax markiert. Tritt nun eine solche markierte Biene in den Lichtkegel, wird ein gewisser Teil des ausgestrahlten Lichts zum impulsgebenden Phototransistor (I) zurückgeworfen und daraufhin ein über mehrere Stufen verstärkter Stromimpuls zum Zeitmarkenschreiber gesendet. Damit Lichtänderungen, die nicht von einer Reflexmarke ausgehen (unmarkierte Bienen, durch das Flugloch eindringendes Licht), nicht zu einer Impulsgebung führen, ist seitlich neben dem Scheinwerfer, außerhalb des Reflexwinkels von Scotchlite, ein zweiter Phototransistor $(K)$ installiert, der solche allgemeinen Helligkeitsschwankungen registriert, durch geeignete Schaltung kompensiert und so eine Impulsgebung verhindert. Da wegen des engen Reflexwinkels das vom Markierungsplättchen ausgehende Licht den Kompensationstransistor nicht trifft, wird in diesem Fall die Wirksamkeit des ersten Transistors nicht aufgehoben. 


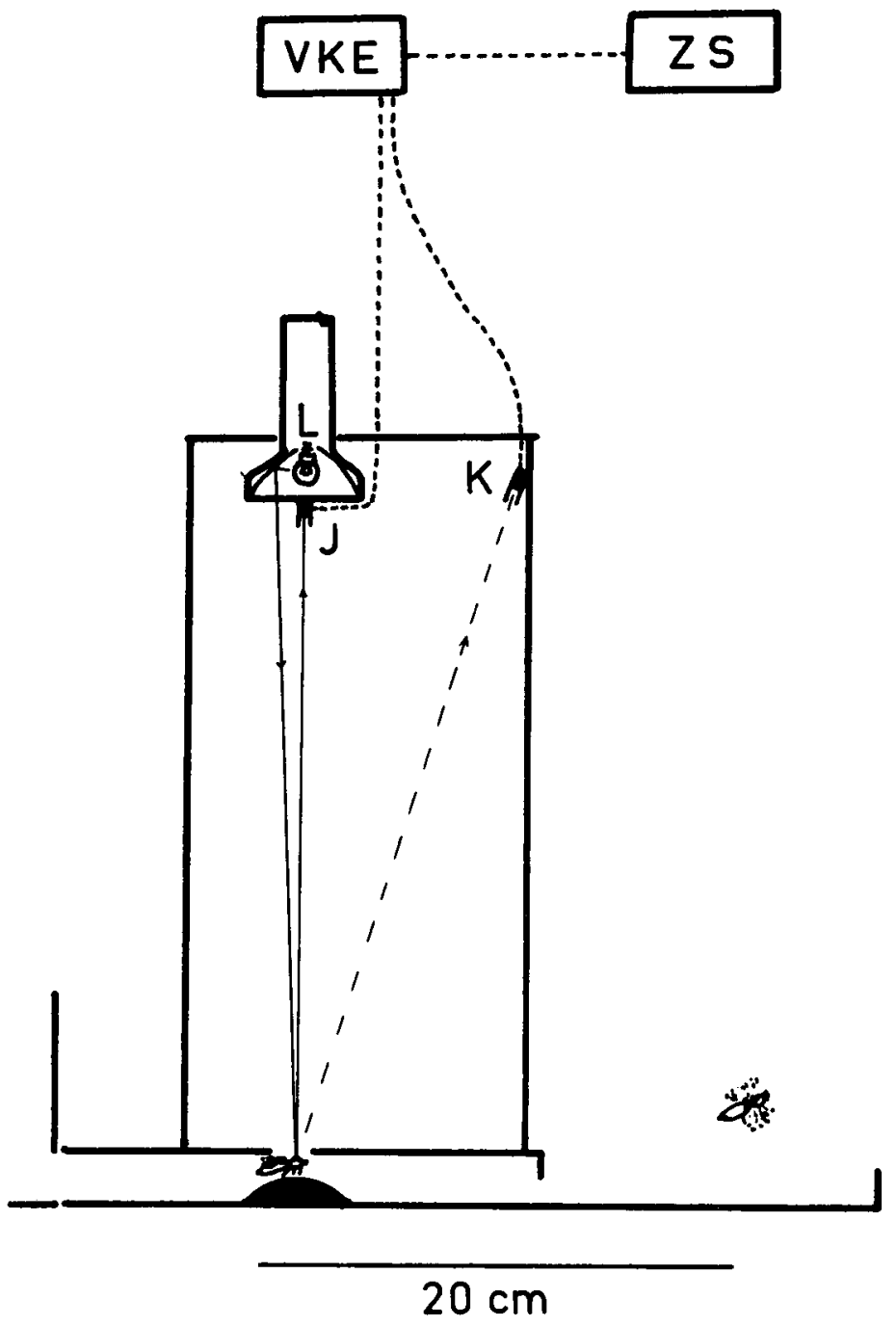

Авв. 1. - Schematische Darstellung der Lichtschleuse und der angeschlossenen Elektronik. J = impulsgebender Phototransistor, $\mathrm{K}=$ Kompensationstransistor, $\mathrm{VKE}=$ Verstärker - und Kompensationselektronik, $\mathrm{ZS}=$ Zeitmarkenschreiber.

Fig. 1. - Représentation schématique du sas lumineux et de l'appareillage êlectronique qui lui est associé. $\mathrm{J}=$ phototransistor générateur d'impulsions. $\mathrm{K}=$ transistor compensateur. VKE $=$ amplificateur ei dispositif électronique de compensation. $\mathrm{ZS}=$ inscripteur.

Der lichtabhängige Teil der Apparatur ist matt-schwarz gestrichen, um Störstrahlen zu vermeiden. Einzelheiten der Schaltung sind Abb. 2 zu entnehmen ${ }^{1}$.

Die “ Bienenpassage » ist von dem die Lichtelektronik beherbergenden

1. Technische Details für den speziell interessierten Leser :

Die Schaltung besteht aus einem empfindlichen Differenzverstärker und einem nachgeschalteten 
Turm durch ein Deckbrett, das ein mit Fliegendraht verschlossenes c Sichtloch ) enthält, abgetrennt. Bei den ersten Vorversuchen zeigte sich, daß die Bienen häufig mit dem Rücken nach unten an diesem Brett entlang liefen, so daß der Phototransistor das Markierungsplättchen nicht « sehen ) konnte.

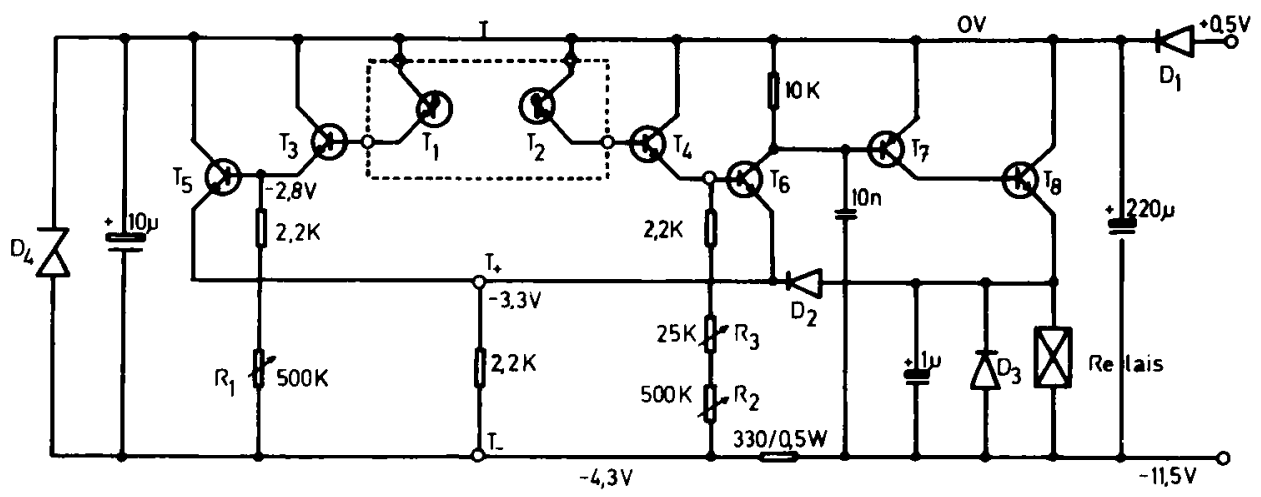

Aвв. 2. - Schaltplan der verwendeten Elektronik. $\mathrm{T}_{2}$ entspricht dem impulsgebenden Phototransistor (J) aus $A$ bb. 1 und $\mathrm{T}_{1}$ dem Kompensationstransistor $(\hat{K})$.

Fıg. 2. - Schéma de montage du dispositif électronique. $\mathrm{T}^{\perp}$ correspond au phototransistor générateur d'impulsions désigné par la lettre $\mathbf{J}$ dans la figure $I$ et $\mathrm{T}_{1}$ au transistor compensateur (K).

Um das zu verhindern, wurde das mit schwarzer Plastikfolie beklebte Deckbrett auf der Unterseite mit Paraffinöl bestrichen.

Die anfangs erwogene Markierung mit Radioisotopen hätte für uns einen größeren technischen Aufwand bedeutet. Vor allem aber bietet die lichtelektronische Úberwachung eine Ausbaumöglichkeit, für die es bei Verwendung

Schaltverstärker mit genau definiertem Arbeitspunkt. Sobald an der Basis von $T_{6}$ eine höhere Spanung liegt als an der Basis von $T_{5}$, schaltet $T_{6}$ und damit $T_{7}$ über $T_{8}$ das Relais. An der Basis von $\mathbf{T}_{5}$ werden mit $R_{1} 2,8 \mathrm{~V}$ eingestellt, an den beiden Testanschlüssen $T_{+}$und $T_{-}$liegt dann $1 \mathrm{~V}$. Darauf wird $R_{2}$ und $R_{3}$ (Feinregelung) so eingestellt, daß das Relais gerade nicht schaltet. Wird nun die Beleuchtung von $T_{1}$ (= Kompensationstransistor) und $\mathrm{T}_{2}(=$ impulsgebender Transistor) gleichzeitig geändert, so ändert sich zwar die gemeinsame Emitterspannung von $\mathrm{T}_{5}$ und $\mathrm{T}_{6}$, aber nicht die Emp findlichkeit, da sich die Spannung an der Basis von $T_{5}$ und $T_{6}$ im gleichen Maße ändert. Das gleiche gilt in weiten Grenzen bei Temperaturund Spannungsschwankungen. Das Relais kann nur ansprechen, wenn die Beleuchtung von $T_{2}$ stärker erhöht wird als die von $T_{1}$. Zur Ausrichtung der Phototransistoren vgl. Abb. 1 .

Technische Daten :

$\mathrm{T}_{1}, \mathrm{~T}_{2}$ : BPY 62 I mit entferntem Basisanschluß

$\mathrm{T}_{3}, \mathrm{~T}_{4}: \mathrm{BC} 108 \mathrm{C}$

$\mathrm{T}_{5}, \mathrm{~T}_{6}: \mathrm{BC} 109 \mathrm{C}$

$\mathrm{T}_{7}: \quad \mathrm{BC} 177 \mathrm{~B}$

$\mathrm{T}_{8}$ : $2 \mathrm{~N} 1711$

$\mathrm{D}_{1}$ : $\quad 1 \mathrm{~N} 4002$

$\mathrm{D}_{2}, \mathrm{D}_{3}:$ 1N 914

$D_{4}: \quad$ ZF 4.3

Relais : $\mathrm{R} \geqslant 150 \Omega$, Ansprechspannung $\leqslant 6 \mathrm{~V}, \mathrm{U}_{\max } \geqslant 12 \mathrm{~V}$, 2 Arbeitskontakte

Alle Kondensatoren $35 \mathrm{~V}$

Widerstände : $330 @ / 0,5 \mathrm{~W}$, alle übrigen $0.1-0.5 \mathrm{~W}$

Stromversorgung: $12 \mathrm{~V} \pm 3 \mathrm{~V} / 100 \mathrm{~mA}$ bei $12 \mathrm{~V}$ und 220 ㅇ Relaisswiderstand

Ruhestrom : $25 \mathrm{~mA}$ bei $12 \mathrm{~V}$

Beleuchtung : 0,1 Lux -10 Lux

Helligkeitskompensation : $\pm 30 \%$ (auch Fremdlicht)

Regelspannung (an den Testpunkten $\mathrm{T}_{+} / \mathrm{T}_{-}$) : $1 \mathrm{~V}$ 
radioaktiver Substanzen (jedenfalls solange man sich mit niedrigen Dosen begnügt) kein Äquivalent gibt : Die Verwendung verschiedenfarbiger Markierungen und entsprechender Filter vor den einzelnen Phototransistoren erlaubt eine getrennte Registrierung verschieden markierter Tiere im selben Stock.

Bei den meisten Fragestellungen ist es erwünscht, daß Ein - und Ausgang getrennt registriert werden. Außerdem ist die individuelle Erfassung numerierter Bienen oft von großem Wert. Eine hohe Aktivität durch wenige markierte Bienen, die in rascher Folge ein - und ausfliegen, ist nicht das selbe wie eine gleich hohe Aktivität, die durch alle markierten Individuen bei längerer Flugdauer bedingt ist. Das erste Problem haben Burrill und Dietz durch ein Klappensystem gelöst. Es ist schwer auszuschließen, daß das Verhalten der Tiere durch den vom Ausgang getrennten Eingang beein flußt wird. Das zweite Problem ist bisher ungelöst. Jedoch arbeiten wir an einem Gerät, das die photoelektronische Technik mit mikrophotographischer Aufzeichnung verbindet und beide Probleme zufriedenstellend lösen soll, ohne daß man Ein - und Ausgang trennen muß. Es wäre demnach möglich, nahezu beliebig viele Tiere automatisch individuell an der “ Passierstelle 》 zu registrieren. Etliche Probleme können auf diese Weise einer Lösung näher gebracht werden.

Vorläufig werden unter Verwendung nur einer Farbe die Aus flüge von Königinnen studiert. Jede der 6 zur Zeit verfügbaren Apparaturen ist mit einer Alarmanlage gekoppelt, so da $\beta$ der in der Nähe befindliche Untersucher sofort auf eine ausfliegende Königin aufmerksam wird. So bleibt bei relativ geringem Zeiteinsatz auch die so wichtige direkte Beobachtung gewährleistet. Die vorläufigen Ergebnisse bestätigen im wesentlichen die moderneren Untersuchungen auf diesem Gebiet. Jede Königin wird in der Regel auf mehreren Aus flügen begattet. Verlassen des Stockes nach Beginn der Eiablage konnten wir bisher nicht beobachten.

Eingegangen im Mai 1974

Reçu pour publication en mai 1974

\section{RÉSUMÉ \\ MÉTHODE D'ENREGISTREMENT DES SORTIES ET DES RENTRÉES D'ABEILLES INDIVIDUELLES}

On décrit une méthode qui permet d'enregistrer automatiquement et sélectivement les sorties et les rentrées d'abeilles individuellement déterminées. Ces abeilles portent sur le thorax une pastille réfléchissante qui agit sur un dispositif photoélectrique qui enregistre les passages sur dérouleur de bande. Une partie du rayonnement provenant de la source lumineuse est renvoyée par la pastille de marquage sur un phototransistor générateur d'impulsions. Un second transistor est placé en dehors de l'angle étroit de réflexion et empêche toute impulsion pouvant provenir des variations générales d'intensité lumineuse, celles par exemple, qui seraient provoquées par le passage d'une abeille non marquée. La méthode a été utilisée jusqu'ici pour l'étude du vol nuptial des reines. 